\title{
COMMISSIONING OF THE CRYOGENIC SYSTEM FOR THE ATLAS SUPERCONDUCTING MAGNETS
}

\author{
N. Delruelle ${ }^{1}$, F. Haug ${ }^{1}$, H. Ten Kate ${ }^{1}$, G. Passardi ${ }^{1}$, R. Pengo ${ }^{1}$, M. Pezzetti ${ }^{1}$, O. Pirotte ${ }^{1}$, \\ E. Baynham ${ }^{2}$, T. Bradshaw ${ }^{2}$, and J. Rochford ${ }^{2}$
}

The paper describes the test results of the helium cryoplant for the superconducting magnets of the ATLAS particle detector at CERN. It consists of two refrigerators used in common by all the magnets and of two proximity cryogenic systems (PCS) interfacing respectively the toroids and the central solenoid. Emphasis is given to the commissioning of the refrigerators: the main unit of $6 \mathrm{~kW}$ equivalent capacity at $4.5 \mathrm{~K}$ and the thermal shield refrigerator providing $20 \mathrm{~kW}$ between $40 \mathrm{~K}$ and $80 \mathrm{~K}$. The first unit is used for refrigeration at $4.5 \mathrm{~K}$ and for the cooling of three sets of $20 \mathrm{kA}$ current leads, while the second one provides, in addition to the $20 \mathrm{~kW}$ refrigeration of the thermal shields, $60 \mathrm{~kW}$ for the cool-down to $100 \mathrm{~K}$ of the 660 ton cold mass of the magnets. The tests, carried out with the equipment in the final underground configuration, are extended to the PCS that includes the large liquid helium centrifugal pumps (each providing $1.2 \mathrm{~kg} / \mathrm{s}$ ) for forced-flow cooling of the magnets and the complex distribution system needed to fulfil their different operating requirements.

1 CERN, Accelerator Technology Department, Geneva, Switzerland

2 Rutherford Appleton Laboratory, Chilton, Didcot, U.K.

Presented at the CEC-ICMC'05 Conference

29 August-2 September 2005, Keystone, Colorado, USA

Administrative Secretariat

AT Department

CERN

CH - 1211 Geneva 23
Geneva, Switzerland

12 January 2006 


\title{
COMMISSIONING OF THE CRYOGENIC SYSTEM FOR THE ATLAS SUPERCONDUCTING MAGNETS
}

\author{
N. Delruelle ${ }^{1}$, F. Haug ${ }^{1}$, H. Ten Kate ${ }^{1}$, G. Passardi ${ }^{1}$, R. Pengo ${ }^{1}$, M. Pezzetti ${ }^{1}$, \\ O. Pirotte ${ }^{1}$, E. Baynham ${ }^{2}$, T. Bradshaw ${ }^{2}$, and J. Rochford ${ }^{2}$ \\ ${ }^{1}$ CERN, Accelerator Technology Department, \\ Geneva, 1211, Switzerland \\ ${ }^{2}$ Rutherford Appleton Laboratory, \\ Chilton, Didcot, OX11 0QX, U.K.
}

\begin{abstract}
The paper describes the test results of the helium cryoplant for the superconducting magnets of the ATLAS particle detector at CERN. It consists of two refrigerators used in common by all the magnets and of two proximity cryogenic systems (PCS) interfacing respectively the toroids and the central solenoid. Emphasis is given to the commissioning of the refrigerators: the main unit of $6 \mathrm{~kW}$ equivalent capacity at $4.5 \mathrm{~K}$ and the thermal shield refrigerator providing $20 \mathrm{~kW}$ between $40 \mathrm{~K}$ and $80 \mathrm{~K}$. The first unit is used for refrigeration at $4.5 \mathrm{~K}$ and for the cooling of three sets of $20 \mathrm{kA}$ current leads, while the second one provides, in addition to the $20 \mathrm{~kW}$ refrigeration of the thermal shields, $60 \mathrm{~kW}$ for the cool-down to $100 \mathrm{~K}$ of the 660 ton cold mass of the magnets. The tests, carried out with the equipment in the final underground configuration, are extended to the PCS that includes the large liquid helium centrifugal pumps (each providing $1.2 \mathrm{~kg} / \mathrm{s}$ ) for forcedflow cooling of the magnets and the complex distribution system needed to fulfill their different operating requirements.
\end{abstract}

KEYWORDS: ATLAS detector, large helium refrigerator, superconducting magnet. PACS: 07.20.Mc

\section{INTRODUCTION}

ATLAS, the general purpose detector for proton-proton collisions at $14 \mathrm{TeV}$, is currently being assembled at collision point 1 of the Large Hadron Collider at CERN. FIGURE 1 presents the July 2005 status of the barrel integration in Point 1 cavern. 


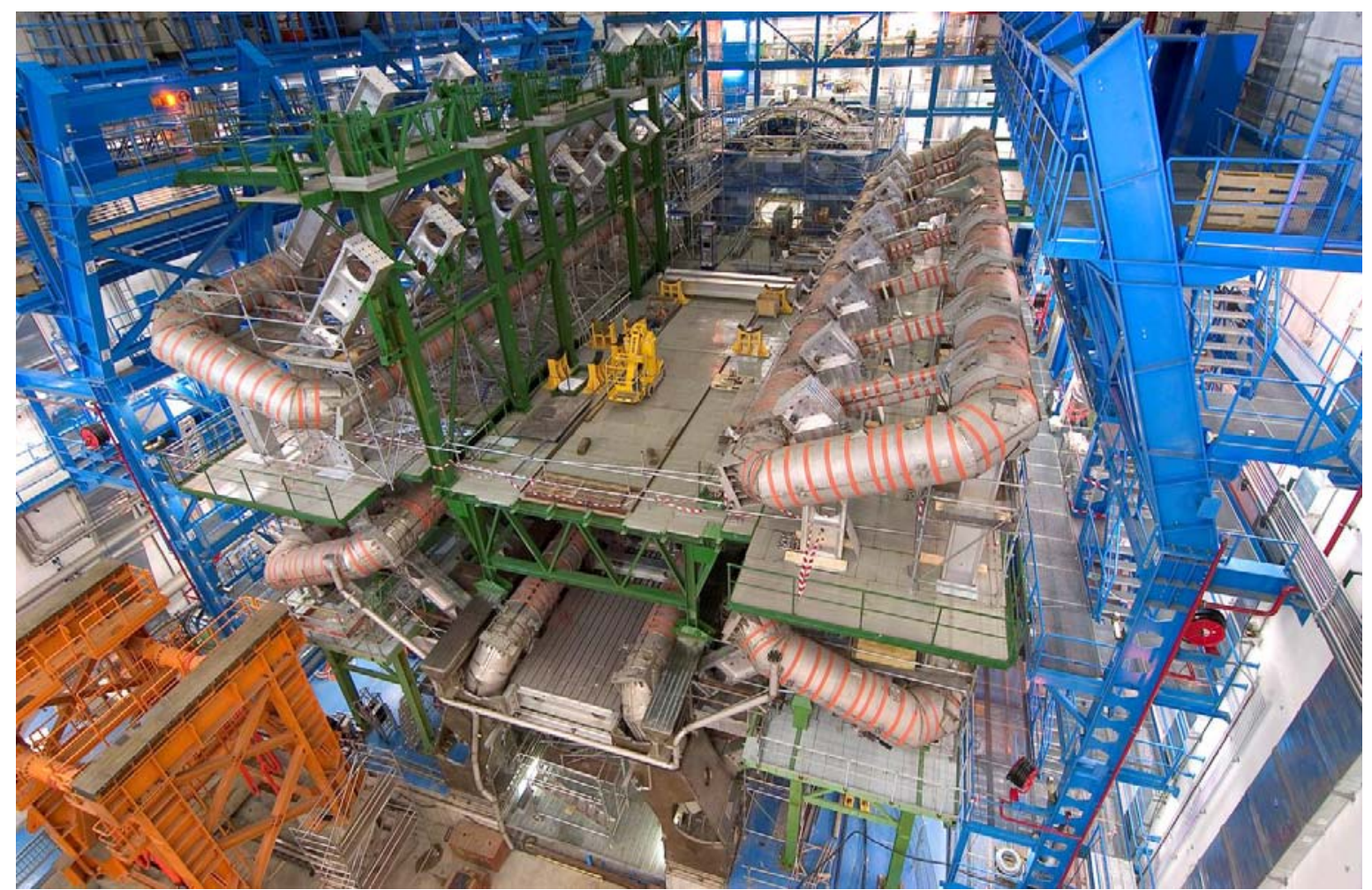

FIGURE 1. July 2005 status of the barrel toroid integration in the ATLAS cavern.

The ATLAS magnet system is based on a thin superconducting central solenoid which generates a uniform longitudinal field for the inner tracker and three superconducting toroid magnets (the barrel and two identical end-caps), each of them made of eight coils producing a tangential field around the central axis for the muon spectrometers [1].

The external envelope of the ATLAS magnets is $20 \mathrm{~m}$ in diameter and $26 \mathrm{~m}$ in length with a total cold mass of 660 tons and a stored energy of $1.6 \mathrm{GJ}$ when magnets are powered up to $20 \mathrm{kA}$.

Two separate helium refrigerators have been installed and are currently being commissioned in a side-cavern close to the detector to allow the four operational modes of magnets; namely, the cool-down from ambient temperature, the steady-state operation at $4.5 \mathrm{~K}$, the thermal recovery (from $\approx 60 \mathrm{~K}$ ) after a fast energy dump and the warm-up [2].

Due to the difference in cooling principle of the central solenoid on the one hand, and the three toroids on the other hand, the helium management towards these four magnets has required two independent Proximity Cryogenic Systems (PCS).

These two PCS, necessary for the operation of each magnet and consisting mainly of current leads cryostat, helium phase-separator, helium centrifugal pumps, distribution valves and safety devices, are thus interfacing the magnets with the two refrigerators.

FIGURE 2 presents a simplified Process Flow Diagram of the helium cryogenic plant for the ATLAS magnets.

\section{HELIUM REFRIGERATORS}

The two helium refrigerators are common to all magnets and provide the required cooling capacity at all temperature levels between $300 \mathrm{~K}$ and $4.5 \mathrm{~K}$.

The main refrigerator (MR), manufactured by Air Liquide in 1991 for an equivalent cooling capacity of $6 \mathrm{~kW}$ at $4.5 \mathrm{~K}$ [3], will be used to cool-down the magnets from $100 \mathrm{~K}$ 


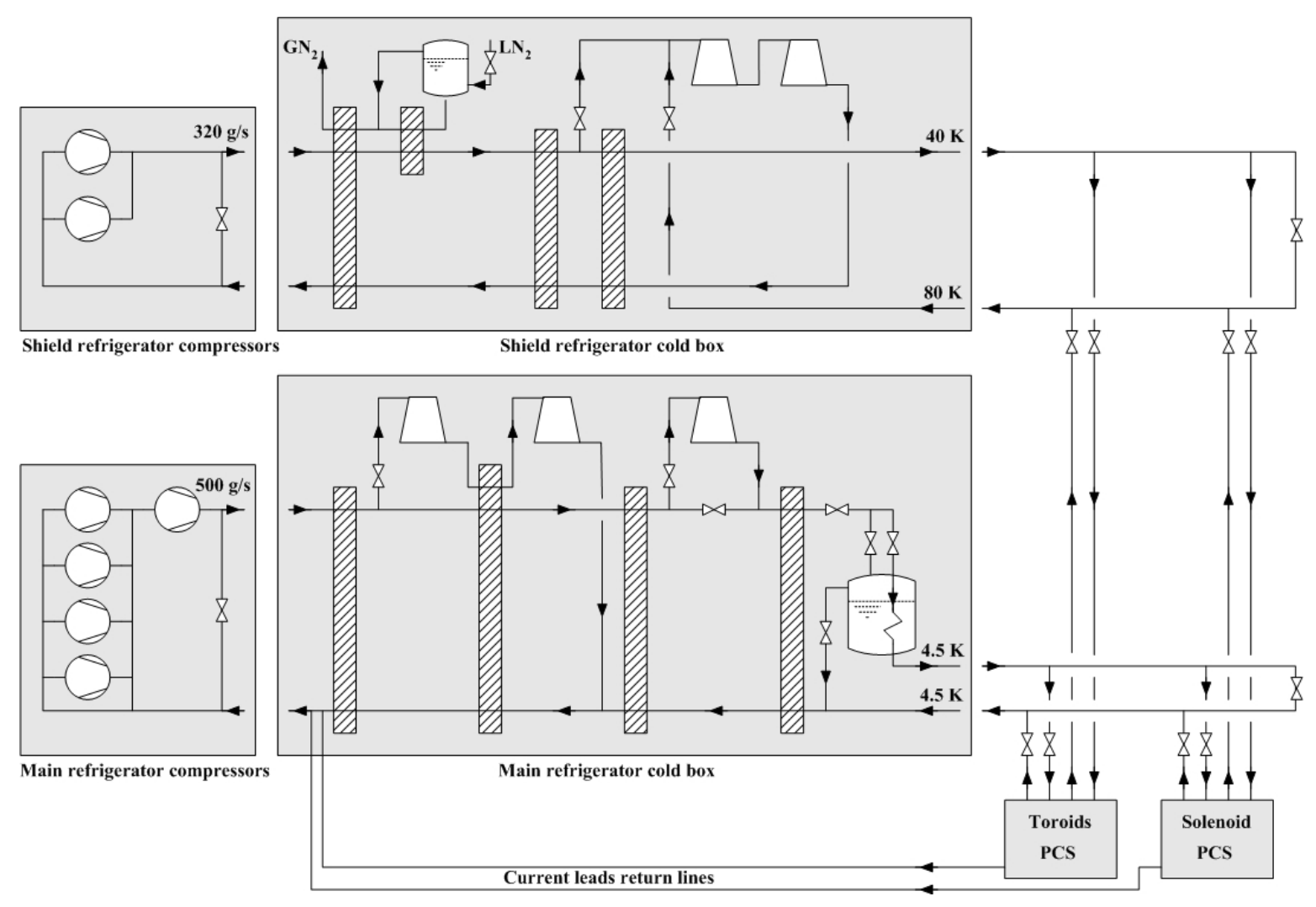

FIGURE 2. Simplified Process Flow Diagram of the helium cryogenic plant.

to $4.5 \mathrm{~K}$ and then ensure the steady-state operation of all magnets at $4.5 \mathrm{~K}$, which includes the cooling of the current leads.

In addition to this machine, a shield refrigerator (SR) manufactured by Linde will be used to cool-down the magnets from $300 \mathrm{~K}$ to $100 \mathrm{~K}$ and then to maintain the thermal shields between $40 \mathrm{~K}$ and $80 \mathrm{~K}$. The SR has been designed to provide two different powers: $20 \mathrm{~kW}$ for the normal shield cooling $(40 \mathrm{~K}-80 \mathrm{~K})$ and $60 \mathrm{~kW}$ when boosted by liquid nitrogen $\left(\mathrm{LN}_{2}\right)$ during the one-month magnet cool-down phase.

\section{Main Refrigerator}

The MR, initially installed at CERN in 1992 for a test station, has been dismounted and re-installed in the ATLAS side-cavern in 2003.

Five cycle screw compressors provide a total mass flow of $0.500 \mathrm{~kg} / \mathrm{s}(500 \mathrm{~g} / \mathrm{s})$. The cycle helium is initially compressed from $0.101 \mathrm{MPa}(1.01 \mathrm{bar})$ up to $0.580 \mathrm{MPa}$ (5.80 bar) by four compressors discharging in parallel into a fifth machine which finally raises the pressure up to $1.940 \mathrm{MPa}$ (19.40 bar).

The refrigerator is based on a two-pressure cycle and equipped with three turbines (FIGURE 2). Two of them, mounted in series, expand the cycle helium from $1.940 \mathrm{MPa}$ (19.40 bar) to $0.120 \mathrm{MPa}(1.20 \mathrm{bar})$. Their discharge temperature is respectively $58 \mathrm{~K}$ and $16 \mathrm{~K}$. The J-T flow is then processed into a third turbine which expands down to $0.260 \mathrm{MPa}(2.60 \mathrm{bar})$ at a discharge temperature of $5.2 \mathrm{~K}$. The final expansion is performed in a J-T valve producing two-phase saturated helium at $0.130 \mathrm{MPa}(1.30 \mathrm{bar})$.

Initially, the control and safety of each turbine was provided by a dedicated Siemens Simatic S5 programmable logic controller (PLC). From a control point of view, these three PLCs were acting independently from the refrigerator's control software and were using a 
non-documented program delivered by the plant manufacturer. Therefore, in order to improve this situation and make easier the turbines operation, we decided to remove these Siemens PLCs and to program in-house the complete control and safety software for the three turbines. Up to now (August 2005), we have been running successfully the turbines for extended periods without any problem and in very stable conditions.

In January 2005, slightly after the start of the cold box commissioning, we had to face with activated charcoal contamination problems in the turbine circuits. The problem was solved by changing the $30 \mu \mathrm{m}$ filters mounted downstream of both $80 \mathrm{~K}$ and $20 \mathrm{~K}$ adsorbers heavily clogged by ten years of previous operation in the test station.

More recently (August 2005), we were obliged to replace the third turbine which jammed during the mechanical adjustment of the gas-bearing supply pressure.

The commissioning and tuning of the cold box is not yet finished (August 2005), but we have already achieved an isothermal refrigeration power of $5.2 \mathrm{~kW}$ at $4.5 \mathrm{~K}$ with a cycle high-pressure of 1.91 MPa (19.1 bar).

\section{Shield Refrigerator}

The new shield refrigerator has been delivered to CERN in 2003.

Two identical screw compressors provide a total mass flow of $0.320 \mathrm{~kg} / \mathrm{s}(320 \mathrm{~g} / \mathrm{s})$. The cycle helium can be compressed in a single stage from $0.101 \mathrm{MPa}(1.01 \mathrm{bar})$ up to $1.80 \mathrm{MPa}(18.0$ bar).

Two different operating scenarios were requested for this refrigerator; namely the normal shield cooling when the detector is in steady-state at $4.5 \mathrm{~K}$ and the magnets cooldown phase when we start from ambient temperature.

In the normal shield cooling, only one compressor providing a mass flow of $0.160 \mathrm{~kg} / \mathrm{s}(160 \mathrm{~g} / \mathrm{s})$ at a discharge pressure of $1.120 \mathrm{MPa}(11.2 \mathrm{bar})$ is sufficient to feed the two turbines mounted in series to provide a cooling capacity of $20 \mathrm{~kW}$ between $40 \mathrm{~K}$ and $80 \mathrm{~K}$ (FIGURE 2). The turbine discharge temperature is respectively $56.5 \mathrm{~K}$ and $39.5 \mathrm{~K}$. Although the turbines were designed to produce $20 \mathrm{~kW}$, we actually measured a cooling capacity of $21 \mathrm{~kW}$, a value which is $5 \%$ higher than the specified value. In this scenario, the second screw compressor is there only for redundancy.

On the contrary, during the magnet cool-down phase from $300 \mathrm{~K}$ to $100 \mathrm{~K}$, a helium forced-flow of $0.300 \mathrm{~kg} / \mathrm{s}(300 \mathrm{~g} / \mathrm{s})$ has to be injected into all magnets. And in order to avoid excessive thermal stresses induced by a large temperature gradient in cold masses, the supply helium temperature has to be maintained constantly $40 \mathrm{~K}$ lower than the temperature of the magnets. As the turbines are not designed to provide the $60 \mathrm{~kW}$ capacity requested during the one-month ATLAS cool-down phase, it has been decided to boost the cold box capacity by means of $\mathrm{LN}_{2}$ during this relatively short period.

During the compressors commissioning, the oil-separator was not working properly; we had too much oil arriving into the three coalescing filters. We were therefore obliged to install in October 2004 a fourth coalescer, just downstream of the oil-separator, in order to achieve the required $100 \mathrm{ppm}$ oil-contamination at the intlet of the final three coalescers.

In order to simulate, during the cold box commissioning, the $60 \mathrm{~kW}$ magnet heat load, an electric heater has been installed in a vacuum-insulated by-pass line at the outlet of the cold box. This $60 \mathrm{~kW}$ heater actually consists of 12 individual $5 \mathrm{~kW}$ elements, on some of which we have encountered isolation problems between the heating element itself and the earth. For this reason, we were able to test up to now (August 2005) the SR with only $40 \mathrm{~kW}$ heating power. As soon as the faulty heaters will be replaced, we are confident that the cold box will reach the specified power of $60 \mathrm{~kW}$. During these two different operating scenarios, all parameters of the refrigerator were very stable. 


\section{PROXIMITY CRYOGENIC SYSTEMS}

The PCS, interfacing the fridges with the magnets, distributes the helium gas and liquid to the different cooling circuits.

Although all magnets will be cooled indirectly by two-phase helium flowing into pipes welded on the cold mass, different flow schemes have been adopted for the three toroids (the barrel and the two end-caps) and for the central solenoid, requiring thus two independent PCS [4].

One centrifugal pump will provide $1.2 \mathrm{~kg} / \mathrm{s}(\approx 10 \mathrm{~L} / \mathrm{s})$ of helium forced flow to keep the toroids at their operating temperature of $4.5 \mathrm{~K}$.

On the contrary, for the central solenoid having a more simple cylindrical structure and piping distribution, both supercritical helium forced-flow provided directly by the main refrigerator at $\approx 0.260 \mathrm{MPa}(2.60$ bar) and thermo-siphon cooling (as back-up) have been designed.

\section{PCS for the Three Toroids}

The proximity system mainly consists of a distribution valve box which contains a helium phase-separator, a current lead cryostat, an 11'000 liter buffer Dewar and a pump cryostat housing two centrifugal pumps. Each pump can provide a mass flow of $1.2 \mathrm{~kg} / \mathrm{s}$ at a nominal pressure head of $40 \mathrm{kPa}$ (400 mbar). A simplified Process Flow Diagram of the PCS is shown in FIGURE 3.

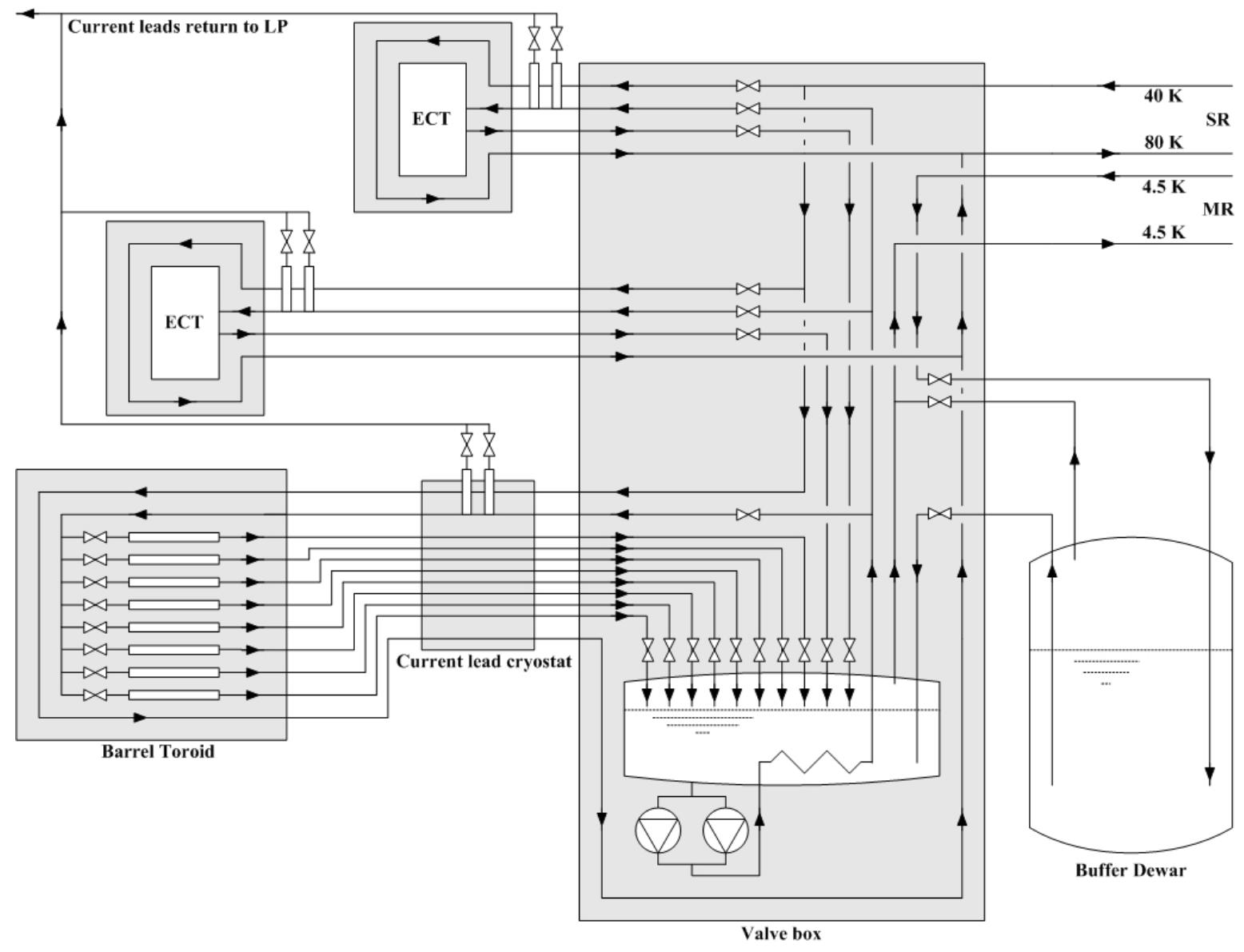

FIGURE 3. Simplified Process Flow Diagram of the toroid PCS. 
When magnets are operated at $4.5 \mathrm{~K}$, the main refrigerator will fill and keep constant the phase-separator level via the buffer Dewar. The saturated helium is then withdrawn at the suction of the centrifugal pump which forces the flow in parallel to both end-caps and to the barrel toroid.

In the eight race-track coils constituting the BT, the helium flow distribution is controlled by eight cryogenic valves and restrictors placed at the entrance of the parallel cooling circuits of each coil, whereas only calibrated orifices equalize the distribution of helium in each ECT.

Part of the liquid helium flowing to the BT and the two ECT's is used for cooling the three pairs of $20 \mathrm{kA}$ current leads required for the magnet powering.

The buffer Dewar, with a capacity of 11'000 liters, has been installed in order to provide enough cooling for a slow discharge $(\approx$ two hours) of the toroids in case of main refrigerator failure.

The most critical parts of the PCS, namely, the three $1.2 \mathrm{~kg} / \mathrm{s}$ centrifugal pumps [5] (two installed in the PCS and one spare) and the $20 \mathrm{kA}$ current leads have been thoroughly tested at the surface before their installation underground. The remaining part of the PCS will be tested in its final underground position in September-October 2005 with helium provided by the two refrigerators and with internal heaters simulating the thermal load of the three toroids.

\section{PCS for the Solenoid}

The proximity cryogenic system for the central solenoid is based on two major components: a 250-liter control Dewar housing the $8 \mathrm{kA}$ current leads and a valve unit equipped with all the instrumentation and the control valves [6].

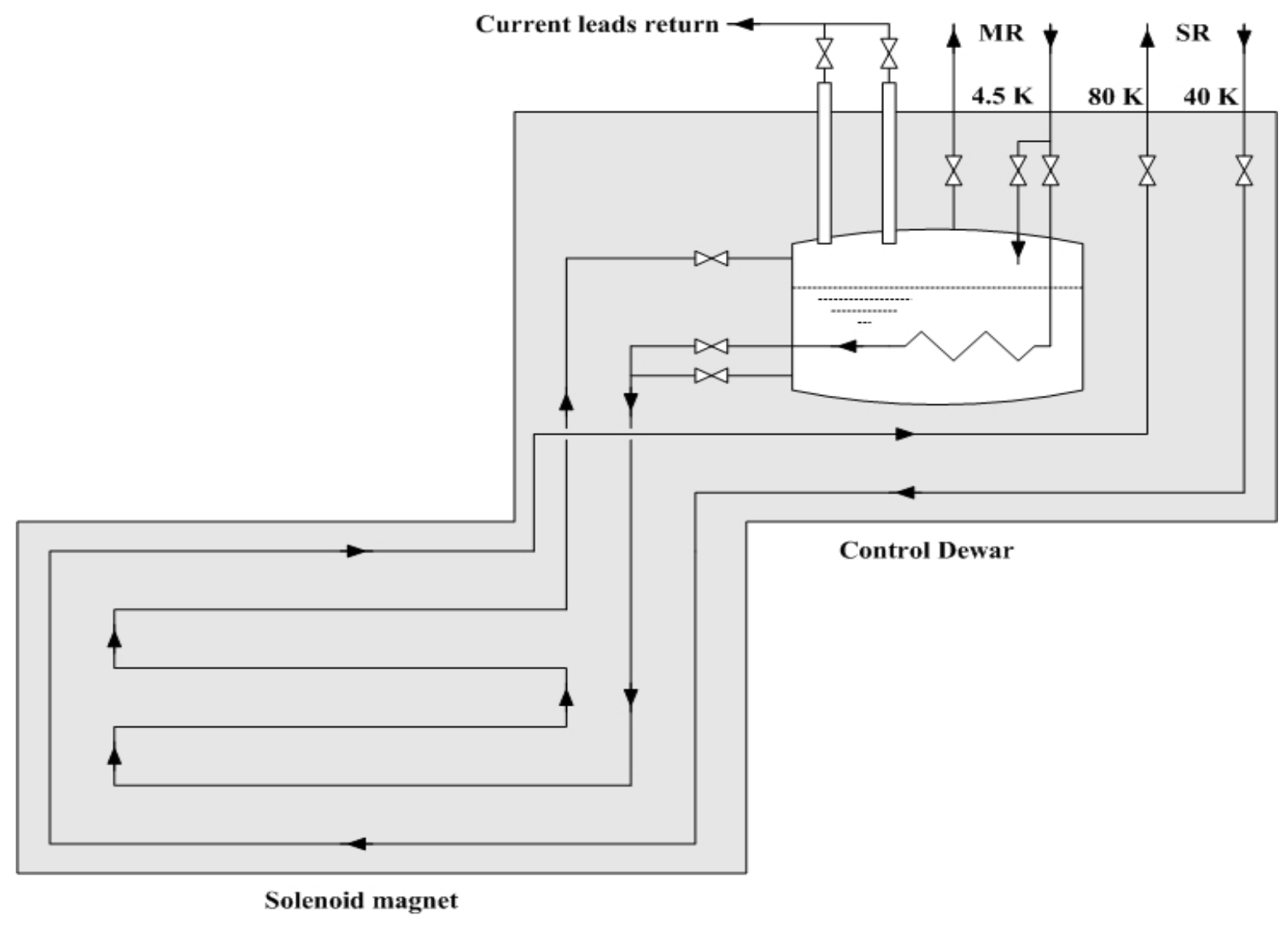

FIGURE 4. Simplified Process Flow Diagram of the solenoid PCS. 
In the final underground installation, the control Dewar will be placed at the top of the ATLAS detector, on a support structure located at a distance of $13 \mathrm{~m}$ from the central axis.

Cryogenic connections between the solenoid coil and the Dewar will be done via a chimney which also houses the superconducting bus bar.

In steady-state operation at $4.5 \mathrm{~K}$, the main refrigerator will provide supercritical helium at $0.260 \mathrm{MPa}(2.60 \mathrm{bar})$ at the PCS interface. The refrigerator flow will be split in two parts. The first one will keep constant the Dewar level while the second one, after passing in a heat exchanger immersed in the Dewar, will be expanded to obtain a twophase cooling of the solenoid cold mass. FIGURE 4 shows a simplified Process Flow Diagram of the PCS.

In case of main refrigerator stop, the 250 liters stored in the Dewar are directly supplied to the magnet in a gravity-assisted thermo-siphon mode, providing then a sufficient autonomy for a slow discharge of the magnet.

Although this PCS was successfully tested at $4.5 \mathrm{~K}$ in Japan with its own control system before shipment to CERN, it was re-commissioned in 2004 in a surface building at CERN, equipped with the final process control, magnet control (for the powering/ discharging of the magnet) and magnet safety (with its hard-wired interlocks) systems.

\section{CONCLUSIONS}

Both helium refrigerators are now (August 2005) nearly commissioned in their final underground side cavern. Maximum cooling capacities in the different operational modes have not all yet been achieved, but we are fully confident to reach these values in the next months.

The performance of the three $1.2 \mathrm{~kg} / \mathrm{s}$ centrifugal pumps and the $20 \mathrm{kA}$ current leads, representing the most critical part of the toroids proximity cryogenic system, have been verified in 2004 on a surface test facility and gave fully satisfactory results. The phaseseparator, the buffer Dewar and the valve box, now installed in the detector cavern, will be tested with the two refrigerators in September-October 2005.

The proximity cryogenic system of the central solenoid as well as its magnet control system and magnet safety system has been thoroughly tested at ground level, with their final PLC control system after the integration of the solenoid in the LAr cryostat. We are now waiting the complete assembly of the barrel toroid to install on its top sector the solenoid control Dewar and the current lead cryostat for the barrel toroid.

The first cool-down of the complete barrel toroid currently being assembled in the ATLAS underground cavern is scheduled for December 2005.

\section{ACKNOWLEDGEMENTS}

Special thanks to G. Cuccuru and S. Carré who provided a valuable technical assistance for the commissioning of these refrigerators.

\section{REFERENCES}

1. ATLAS Magnet System: Technical Design Report, CERN/LHCC/97-18, April 1997.

2. Delruelle N., Haug F., Passardi G., Ten Kate H., "The Helium Cryogenic System for the ATLAS Experiment", in IEEE Transactions on Applied Superconductivity, Vol. 10, No. 1, March 2000, pp. 1511-1513. 
3. Gistau G. M., Bonneton M., “A $6 \mathrm{~kW}$ at $4.5 \mathrm{~K}$ Helium Refrigerator for CERN's Cryogenic Test Station”, in Advances in Cryogenic Engineering 39A, edited by P. Kittel, Plenum, New York, 1994, pp. 547-551.

4. Haug F., Passardi G., Pengo R., Ten Kate H., Baynham E., Bradshaw T., Brown G., Cragg D., Crook M., Orlowska A. H., Rochford J., Sole D., “The Proximity Cryogenic System for the ATLAS Toroidal Magnets", in Advances in Cryogenic Engineering 47A, edited by S. Breon et al., Plenum, New York, 2002, pp. 155-160.

5. Pengo R., Junker S., Passardi G., Pirotte O., Ten Kate H., "Tests Results of a 1.2 kg/s Centrifugal Liquid Helium Pump for the ATLAS Superconducting Toroid Magnet System", in Proceedings of the 19th International Cryogenic Engineering Conference (ICEC19), edited by G. Gistau and P. Seyfert, Narosa Publishing House, 2003, pp. 71-74.

6. Doi Y., Haruyama T., Kawai M., Konda T., Kondo Y., Makida Y., Yamamoto A., Haug F., Metselaar J., Passardi G., Pavlov O., Pezzetti M., Pirotte O., Ruber R., Sbrissa E., Ten Kate H., Tyrvainen H., "Final Testing of the ATLAS Central Solenoid Before Installation", presented at the 20th International Cryogenic Engineering Conference (ICEC20), May 2004, Beijing, China, to be published. 